\title{
EL DIÁLOGO INTERRELIGIOSO EN EL ESPACIO PÚBLICO: RETOS PARA LOS AGENTES SOCIOEDUCATIVOS EN CATALUÑ̃'
}

\author{
INTERRELIGIOUS DIALOGUE IN THE PUBLIC SPHERE: CHALLENGES \\ FOR SOCIOEDUCATIONAL ACTORS IN CATALONIA \\ DIÁLOGO INTER-RELIGIOSO NO ESPAÇO PÚBLICO: DESAFIOS PARA \\ OS AGENTES SOCIOEDUCACIONAIS NA CATALUNHA
}

\author{
Marta SABARIEGO PUIG*, Ruth VILÀ BAÑOS* \& Montserrat FREIXA NIELLA* \\ *Universidad de Barcelona
}

\begin{tabular}{|c|c|}
\hline & $\begin{array}{r}\text { Fecha de recepción del artículo: } 25 . X I .2018 \\
\text { Fecha de revisión del artículo: } 3.1 \mathrm{~V} .2018 \\
\text { Fecha de aceptación final: } 24 \text {.VI.2018 }\end{array}$ \\
\hline $\begin{array}{l}\text { PALABRAS CLAVE: } \\
\text { Diversidad cultural } \\
\text { estudios de } \\
\text { religiones } \\
\text { grupos religiosos y } \\
\text { culturales } \\
\text { inmigración } \\
\text { actitudes sociales } \\
\text { entorno social }\end{array}$ & $\begin{array}{l}\text { RESUMEN: Este artículo examina el concepto y los elementos que favorecen el diálogo in- } \\
\text { tercultural e interreligioso, desde el punto de vista de expertos implicados en la gestión de } \\
\text { la diversidad cultural en el espacio público: profesionales de la Administración, entidades } \\
\text { sociales y mundo académico en Cataluña. Responde a un estudio descriptivo-comprensivo } \\
\text { que sigue un enfoque cualitativo, y en el que se ha optado por la fundamentación hermenéu- } \\
\text { tica-interpretativa para obtener el relato de estos tres colectivos. Se han desarrollado once } \\
\text { entrevistas semiestructuradas a participantes representativos de cada uno de ellos. Los resul- } \\
\text { tados revelan definiciones diferenciadas pero complementarias sobre el diálogo interreligio- } \\
\text { so: relación entre cultura y religión, diálogo como un primer paso de reconocimiento mutuo } \\
\text { y los beneficios de éste. Los participantes aportan elementos que facilitan este diálogo. Se } \\
\text { concluye con un modelo de gestión de la diversidad religiosa en el espacio público con pro- } \\
\text { puestas comunitarias y educativas para orientar futuras políticas de prevención, mediación y } \\
\text { cohesión social en Cataluña. }\end{array}$ \\
\hline
\end{tabular}

CONTACTO CON LOS AUTORES: MARTA SABARIEGO PUIG. Universidad de Barcelona. Facultad de Educación. Departamento Métodos de Investigación y Diagnóstico en Educación. Despacho 202. Passeig de la Vall d'Hebron, 171. Edifici Llevant-2a planta. O8035 Barcelona. Tel.: (o034) 93.403.50.10. E-mail: msabariego@ub.edu 


\begin{tabular}{|c|c|}
\hline $\begin{array}{l}\text { KEY WORDS: } \\
\text { cultural differences } \\
\text { religion studies } \\
\text { religious cultural } \\
\text { groups } \\
\text { immigration } \\
\text { social attitudes } \\
\text { social environment }\end{array}$ & $\begin{array}{l}\text { ABSTRACT: This article examines the concept and the elements that encourage intercultural } \\
\text { and interreligious dialogue, from the point of view of experts involved in the management } \\
\text { of religious diversity in the public space: policy-makers, associations and academics in Cata- } \\
\text { lonia. It responds to a comprehensive-descriptive study with a qualitative approach and an } \\
\text { hermeneutic-interpretative rationale as the most appropriate methodology to examine the } \\
\text { accounts of the three aforementioned groups. Eleven individual semi-structured interviews } \\
\text { have been conducted with representatives of each group. The results reveal different but } \\
\text { complementary definitions of interreligious dialogue: the relationship between culture and } \\
\text { religion, dialogue as a first step of mutual recognition and the benefits of it. The participants } \\
\text { contribute elements that facilitate this dialogue. It concludes with a management model of } \\
\text { religious diversity in the public space with community and educational proposals to guide } \\
\text { future prevention, mediation and social cohesion policies in Catalonia. }\end{array}$ \\
\hline $\begin{array}{l}\text { PALAVRAS-CHAVE: } \\
\text { diversidade cultural } \\
\text { estudos de religiões } \\
\text { grupos religiosos e } \\
\text { culturais } \\
\text { imigração } \\
\text { atitudes sociais } \\
\text { ambiente social }\end{array}$ & $\begin{array}{l}\text { RESUMO: Este artigo examina o conceito e os elementos que favorecem o diálogo intercultu- } \\
\text { ral e interreligioso, do ponto de vista de especialistas envolvidos na gestão da diversidade cul- } \\
\text { tural no espaço público: profissionais da Administração, entidades sociais e o mundo acadê- } \\
\text { mico da Catalunha. Responde a um estudo descritivo-abrangente que segue uma abordagem } \\
\text { qualitativa, e no qual a base hermenêutico-interpretativa foi escolhida para obter a explicação } \\
\text { desses três grupos. Onze entrevistas semi-estruturadas foram desenvolvidas para os parti- } \\
\text { cipantes representativos de cada uma delas. Os resultados revelam definições diferentes } \\
\text { mas complementares do diálogo inter-religioso: a relação entre cultura e religião, o diálogo } \\
\text { como primeiro passo para o reconhecimento mútuo e os benefícios dele. Os participantes } \\
\text { contribuem com elementos que facilitam esse diálogo. Conclui com um modelo de gestão } \\
\text { da diversidade religiosa no espaço público com propostas comunitárias e educacionais para } \\
\text { orientar futuras políticas de prevenção, mediação e coesão social na Catalunha. }\end{array}$ \\
\hline
\end{tabular}

\section{Introducción}

Las distintas tradiciones religiosas siempre han estado en contacto, pero las miradas mutuas de las religiones han sido excluyentes y su rivalidad ha causado muchos conflictos y enfrentamientos sociales (Javier, 2014). En la Europa actual, el contacto entre religiones ha aumentado por la inmigración, haciendo más compleja la presencia de la religión en el espacio público y confirmando el vaticinio que ya avanzó Kettel (2009): "el debate sobre el papel de la religión en la esfera pública parece ser uno de los temas que definen el siglo XXI" (p. 1). Efectivamente, los conceptos relacionados con la religión despiertan discusión y controversia, generando emociones y sentimientos de alta intensidad (Brie, 2011).

Hozu y Frunza (2013) argumentan dos principios que fundamentan por qué es necesario el debate del hecho religioso en un espacio público moderno caracterizado por la secularización: primero, el auge de los procesos de radicalización en los movimientos de globalización hacen que sea necesario reevaluar el ajuste de la religión en el espacio público de forma no violenta; y segundo, hay que prestar la atención necesaria a las relaciones de ambigüedad entre los estados y las organizaciones religiosas. Aaroe (2012) identifica dos visiones sobre la relación del estado con la religión/es: la religión debería formar parte de la cultura pública secular; y los que no lo piensan así. Su investigación pone de manifiesto que ambas posturas tienden a la intolerancia religiosa, aunque por motivos diferentes: la primera visión, por proyectar estereotipos al resto de religiones no mayoritarias, y la segunda por el principio de secularidad que no siempre es respetado en la práctica: a menudo los estados invocan la defensa de la laicidad de forma discrecional para otorgar más o menos derechos a diversas confesiones (Bowen, 2008). Ante esta situación, la diversidad religiosa se manifiesta en el espacio público haciendo emerger el conflicto como en la construcción de lugares de culto o en los símbolos religiosos.

Ante esta realidad se impone la necesidad de un diálogo interreligioso que debería tender a superar tales enfrentamientos. Prieto (2014), haciéndose eco de esta sensibilidad, expone que las distintas tradiciones culturales y religiosas del mundo empiezan a aceptar el pluralismo religioso para reforzar la identidad propia y afirmar la presencia religiosa en la sociedad secular. Por su parte, Dupuis (2002) y más recientemente Tamayo (2012) muestran interés para acercar el pluralismo religioso o la teología del pluralismo religioso a la sociedad. Se trata de una reflexión teológica en un contexto interreligioso sobre el diálogo y en el diálogo, iniciado a partir de la teología cristiana y católica que lleva a la valoración de las religiones en sí mismas.

\section{El diálogo interreligioso: aportaciones para la cohesión social, la prevención y mediación en situaciones conflictivas}

El Libro Blanco sobre el Diálogo Intercultural de la Unión Europea (2006) define el diálogo como un proceso que implica el intercambio respetuoso y 
abierto de puntos de vista entre individuos y grupos de diferentes procedencias y con legados culturales, religiosos y lingüísticos diferentes, sobre la base del mutuo entendimiento y respeto. Los principios del diálogo interreligioso se orientan a la capacidad de escucha y respeto a la diversidad de creencias para identificar la experiencia religiosa común y la apertura a la diferencia desde el ejercicio de una ciudadanía crítica y participativa (Torradeflot, 2012). Ello supone la predisposición a repensar las ideas propias a la luz de las de los demás para el enriquecimiento y transformación (Santiago \& Corpas, 2012).

Esta perspectiva supone un gran reto para el diálogo interreligioso, ya que no parte de una perspectiva excluyente y preeminente de una religión sobre la otra. El convencimiento de la superioridad de una religión por encima de las demás y de otras creencias puede provocar el odio, la exclusión o la persecución manifestándose en incidentes violentos o extremadamente violentos. El diálogo debe buscar la superación de estas barreras (Tamayo, 2005; Torradeflot, 2011). La prevención y mediación interreligiosa de conflictos sirve también para evitar la xenofobia, el racismo y las fobias contra las religiones, especialmente el antisemitismo, la islamofobia y la cristianofobia. De esta forma, el diálogo interreligioso no es un intercambio de cortesía, tradiciones o monólogos; implica "un encuentro en la profundidad y la riqueza de la vida religiosa de la humanidad" (Torradeflot, 2011, p. 5) entre personas o colectivos con legados culturales, religiosos, lingüísticos diferentes para que las religiones se conozcan y se respeten mutuamente. El diálogo intercultural e interreligioso implica la escucha receptiva, activa y recíproca y el testimonio honesto, el respeto y la igualdad (UNESCO, 2015).

Se pueden definir dos tipos de diálogo interreligioso: el primero informal y el segundo organizado (Torradeflot et al., 2012). El primero es el diálogo de vida que se produce en las distintas situaciones de convivencia en la pluralidad. Así, personas de confesiones y creencias diferentes, viven y trabajan juntas compartiendo una vida y unos espacios comunes: las tiendas, el parque, el patio de la escuela, las actividades extraescolares, el café del trabajo, etc. Lo fundamental de este nivel es el respeto mutuo, que en algunos casos puede llegar a la amistad. Son diálogos invisibles, que pasan desapercibidos y no son conscientemente religiosos, pero refuerzan las relaciones humanas.

El diálogo organizado surge por iniciativa de la sociedad civil, las distintas comunidades religiosas o las administraciones municipales. Desde la ciudadanía, el objetivo es tener un mejor y mayor conocimiento mutuo a través de la organización de alguna actividad intercultural o social en el contexto más cercano. Las comunidades religiosas, por su parte, pretenden el encuentro y el diálogo interreligioso para elucidar los valores positivos y velar por las tradiciones, intereses y prioridades comunes de las distintas confesiones. Las administraciones fomentan iniciativas interreligiosas para la cohesión social a partir de la implicación de las comunidades religiosas, especialmente en los barrios o ciudades con gran diversidad cultural y religiosa con posibilidad de conflicto social.

Torradeflot (2011) propone la siguiente clasificación de grupos interreligiosos organizados según su naturaleza y objetivo principal: reconocimiento mutuo, estudio e investigación, profundización e intercambio espiritual, cohesión social y mediación, y representación institucional.

El grupo de reconocimiento mutuo tiene por objetivo el conocimiento mutuo a través del diálogo en una relación interpersonal para descubrir la identidad del otro. Esta situación puede ser el inicio de una amistad y permite tejer relaciones entre los distintos miembros. Son grupos abiertos a todas las personas independientemente de sus religiones o convicciones. Se suelen convocar reuniones presenciales, pero también se organizan comidas compartidas y eventos interreligiosos. Su finalidad es superar la desconfianza y también la posible hostilidad.

El grupo de estudio e investigación incluye expertos, académicos y universitarios juntamente con creyentes de diversas tradiciones para intercambiar las bases teológico-filosóficas de las distintas confesiones religiosas. Se trata de llegar a una comprensión común de la forma en que cada tradición religiosa explica y enfoca la realidad. Pueden ser grupos monotemáticos sobre la paz, el papel de la mujer en las religiones, inmigración, etc. Contribuyen a eliminar prejuicios y falsas ideas ampliando los temas abordados.

En el grupo de diálogo espiritual, las personas creyentes, espirituales o místicas tratan de encontrarse los unos a los otros para exponerse recíprocamente su vida espiritual y de culto. Estos diálogos adoptan la forma de participación en las prácticas de meditación o silencio y de formación mutua por encima del debate y la acción comuna. Según Pannikar (2005) es un diálogo especialmente profundo, conciliador y que inspira otras formas de diálogo.

Los grupos de cohesión social y mediación se encuentran en los municipios y los barrios con gran diversidad y están formados por miembros de organizaciones de la sociedad civil y profesionales de los servicios sociales, educativos, sanitarios municipales. Su objetivo es la cohesión y la 
convivencia, la lucha por la paz y contra la discriminación, y la defensa de los derechos y de los colectivos desfavorecidos.

Finalmente, los grupos de representación institucional están formados por autoridades de tradiciones religiosas que son la voz oficial de sus comunidades y que se constituyen como consejos, foros o asambleas. Su objetivo es tener una voz conjunta y liderar el diálogo de sus respectivas comunidades de fe. Suelen ser los grupos de interlocución con las administraciones.

El diálogo interreligioso de proximidad es el más cercano a la realidad cotidiana revirtiendo, por lo tanto, de forma más eficaz en el bienestar común de la ciudadanía. Puede, además, promover y enriquecer la participación ciudadana, prevenir y mediar en situaciones conflictivas y trabajar para la solidaridad y la justicia (Torradeflot, 2012).

\section{Metodología}

Este artículo corresponde a la segunda fase de un proyecto más amplio (Direcció General d'Afers Religiosos-Ref.2014RELIGoo019) con un diseño metodológico mixto (Creswell, 2003; Tashakkori \& Teddie, 2010) y exploratorio secuencial (Creswell \& Plano, 2011). En esta segunda fase de la investigación se pretende matizar las necesidades normativas detectadas en la fase anterior para la gestión de la diversidad religiosa en el espacio público, basada en el diálogo intercultural e interreligioso, tomando como marco de comprensión el punto de vista de tres agentes socioeducativos: los profesionales de la Administración, las entidades y el mundo académico. Concretamente, los objetivos de este artículo son:
- Comprender el concepto de diálogo intercultural e interreligioso que tienen estos agentes socioeducativos

- Describir los elementos que favorecen el diálogo intercultural e interreligioso según estos agentes socioeducativos

- Identificar y proponer iniciativas comunitarias y/o políticas para favorecer el diálogo intercultural e interreligioso.

Se ha procedido a través de un estudio cualitativo de corte descriptivo-comprensivo para describir e interpretar esta experiencia en los contextos reales (Maykut \& Morehouse, 1999), mediante once entrevistas semi-estructuradas e individuales a profesionales procedentes de los tres perfiles identificados. Esta finalidad justifica la fundamentación hermenéutica-interpretativa para obtener el relato de los profesionales anteriores alrededor de la definición y los elementos que favorecen el diálogo interreligioso e intercultural en el espacio público, desde sus experiencias vitales y el sentido que los hechos tienen para ellos.

\section{Participantes}

Se seleccionaron 11 participantes de forma intencional a partir de criterios como su relevancia y calidad de expertos y agentes sociales implicados en la gestión de la diversidad cultural en el espacio público. Se trata de tres profesionales del ámbito de la administración, seis del ámbito académico y dos procedentes de las entidades. La recogida de datos se realizó entre marzo y abril de 2015. La Tabla 1 resume el perfil profesional de los participantes entrevistados. 
Tabla 1. Perfil profesional de los participantes entrevistados

\begin{tabular}{|c|l|l|}
\hline \multicolumn{1}{|c|}{ Personas } & \multicolumn{1}{|c|}{ Ámbito } & \multicolumn{1}{|c|}{ Profesión } \\
\hline Participante 1 & Entidades & Migrastudium \\
\hline Participante 2 & Académico & Profesor universitario, filósofo y teólogo \\
\hline Participante 3 & Académico & Profesor de la Facultad de Teología de Catalunya \\
\hline Participante 4 & Académico & Profesora del Centre d'Estudi de les Tradicions de Saviesa (CETR). \\
\hline Participante 5 & Administración & $\begin{array}{l}\text { Direcció General d'Afers Religiosos. Departament de Governació i Relacions Institucionals. } \\
\text { Generalitat de Catalunya }\end{array}$ \\
\hline Participante 6 & Administración & Unesco para el Diálogo Interreligioso - UNESCOCAT. \\
\hline Participante 7 & Académico & Profesor de la Universitat Autònoma de Barcelona \\
\hline Participante 8 & Académico & Profesor de la Universitat Pompeu Fabra \\
\hline Participante 9 & Administración & Oficina d'Afers Religiosos. Regidoria de Dona i Drets Civils. Ajuntament de Barcelona \\
\hline Participante 10 & Académico & Profesor de la Universitat Abat Oliva \\
\hline Particicipante 11 & Entidades & Junta Islàmica Catalana \\
\hline
\end{tabular}

\section{Técnicas y procedimiento}

Mediante la técnica de la entrevista semiestructurada individual (Cabrera, 2011) se procedió al registro audio del discurso con el fin de obtener datos directos. A través de 20 preguntas que seguían una secuencia lógica -desde las sociodemográficas, introductorias y básicas- se exploraron cinco dimensiones principales de contenido: i) la aproximación conceptual: espiritualidad y religiosidad; ii) la confesionalidad de los estados laicos; iii) el fomento del diálogo interreligioso; iv) la necesidad de una gestión de la diversidad religiosa ante la discriminación, patriarcado y conflicto interreligioso; y v) las acciones, iniciativas y mejoras desde la educación. En este artículo se presenta el discurso de estos agentes socioeducativos sobre las dimensiones iii y v: el concepto y el fomento del diálogo interreligioso, y la propuesta de acciones, iniciativas para la gestión de la diversidad religiosa para el fomento del diálogo intercultural e interreligioso en el espacio público, a modo de aportaciones en las conclusiones.

\section{Análisis de datos}

Se realizó un análisis cualitativo de la información extraída en las entrevistas con el soporte del programa informático QSRNVIVO 11 para agilizar actividades como la segmentación del texto en citas, la codificación o la escritura de comentarios (Gibbs, 2012).

Posteriormente a la transcripción literal de las entrevistas, se procedió a un análisis cualitativo, dividido en 3 fases:

1. Fase de codificación. Se hizo una lectura de las transcripciones para generar las primeras codificaciones sobre el texto y agruparlas en categorías. El proceso comenzó con la definición de categorías e indicadores que se derivaron deductivamente de conceptos teóricos y trabajos previamente publicados (Augé 2001; Gómez, Alonso \& Cabeza 2011; Henry 2001). La primera lectura de los manifiestos fue realizada por el equipo investigador.

2. Fase de triangulación de categorías. Se realizó un consenso entre los investigadores del equipo sobre las categorías de análisis. Este procedimiento de categorización siguió un doble proceso: inductivo, ya que a partir de las transcripciones se desarrollaron las categorías iniciales, subcategorías y relaciones entre ellas; $y$ un proceso deductivo, pues éstas se examinaron considerando las necesidades normativas identificadas en el marco teórico de referencia durante la fase anterior del proyecto (Álvarez \& Essomba, 2012; Corbí, 2007; Dietz, 2008; Melloni, 2003; Pániker, 2010; Torradeflot, 2012; UNESCO, 2005). El análisis documental llevado a cabo en esta 
primera fase permitió la identificación de cuatro grandes bloques de necesidades normativas para la gestión de la diversidad religiosa (Vilà et al., 2015):

- Necesidad de una clarificación conceptual y la confesionalidad de los estados laicos.

- Necesidad de una gestión de la diversidad religiosa: discriminación, patriarcado y conflicto interreligioso.

- Fomentar el diálogo intercultural e interreligioso.
- Necesidad de acciones, iniciativas y mejoras desde la educación.

Con este referente, una vez establecido un primer sistema provisional de categorías y subcategorías, el equipo de investigación codificó una pequeña muestra de textos individualmente. Esto fue seguido por un análisis grupal que resultó en la reestructuración y el consenso del siguiente sistema de categorías definitivo para codificar todas las unidades de análisis de las entrevistas:

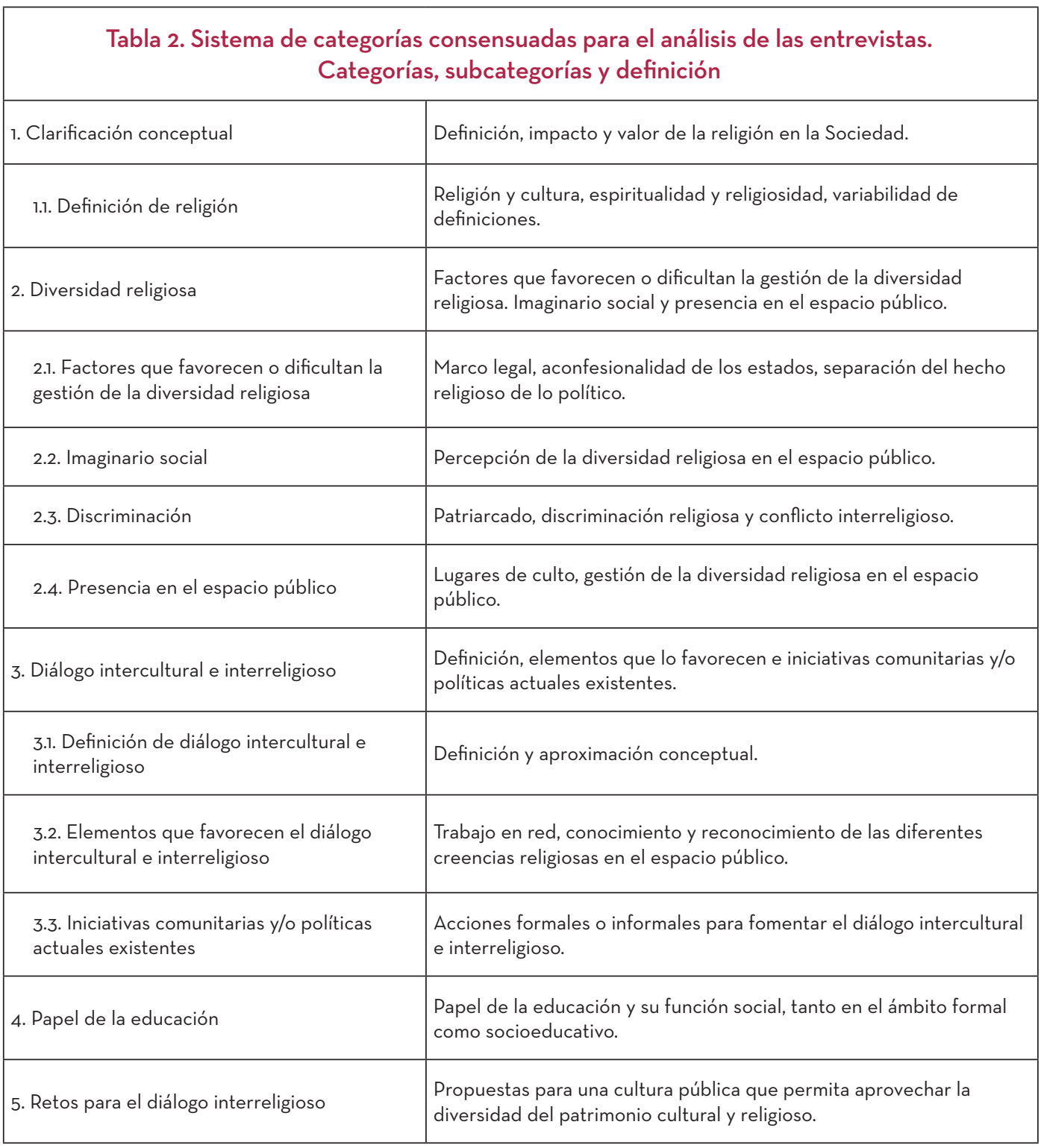

En este artículo se presentan los resultados obtenidos más específicamente en la tercera categoría sobre el diálogo intercultural e interreligioso.

3. Fase de obtención y verificación de resultados. Se realizó un análisis de contenido para la profundización secuencial de la información.
Para cada categoría se analizó la información obtenida a un doble nivel: a) de carácter general, para obtener una visión global de las categorías especificadas, y b) por perfiles de profesionales entrevistados, para matizar los datos en cada uno de los tres colectivos estudiados. 
El artículo concluye con una propuesta de iniciativas comunitarias y/o acciones concretas formales o informales para fomentar el dialogo y favorecer su gestión en el espacio público. Es importante señalar que, si bien se trata de entrevistas a sujetos particulares, el análisis busca una dimensión transversal en el discurso de los colectivos estudiados sin pretender representar o saturar la totalidad de las opiniones de los colectivos a los que hacen referencia (las entidades sociales, la Administración y el mundo académico) en el contexto de Cataluña. Por este motivo, se realiza una triangulación horizontal y de perspectivas cuyo propósito es cruzar las vivencias y el relato de los tres colectivos analizados, poniendo atención tanto a los puntos en común como a las divergencias en el concepto y el diálogo interreligioso e intercultural en el contexto catalán desde esta triple perspectiva.

\section{Resultados}

\section{Definición del diálogo interreligioso e intercultural}

Las narrativas de los tres puntos de vista apuntan a una conceptualización sobre el diálogo intercultural e interreligioso diferenciada. Tal y como se observa en la siguiente matriz de codificación, el porcentaje del peso de palabras codificadas por esta categoría (porcentaje de columna 3.1) no tiene la misma relevancia en todos los colectivos: pesa mucho más en el relato de las personas del mundo académico que en la Administración y las entidades.

\begin{tabular}{|l|c|c|c|}
\hline \multicolumn{4}{|c|}{ Tabla 3. Matriz de codificación comparativa de las categorías de análisis utilizadas } \\
(porcentaje de columna)
\end{tabular}

El análisis más detallado de las palabras más frecuentes y de su significado en el contexto de las entrevistas permite detectar diferentes patrones en la definición del diálogo interreligioso e intercultural aportada por cada uno de los tres colectivos.

La narrativa de los expertos del mundo académico nos acerca a la idea del diálogo interreligioso como aquel que existe entre personas (de tradiciones religiosas diferentes), enfatizando en plano individual, que por necesidad se acercan e intercambian respetuosamente sus creencias. Parafraseando a Pannikar (2003:20) "el diálogo debe producirse a todos los niveles, pero especialmente en el sentido místico entre las raíces espirituales de cada tradición e identidad".

Existen diferentes niveles de diálogo interreligioso (desde los más institucionales hasta los de base ciudadana y social) y, como se ilustra en la marca de nube (Gráfico 1), en el relato aparecen matizados por su finalidad: el reconocimiento del otro y la síntesis que supone cambio, transformación y desarrollo.

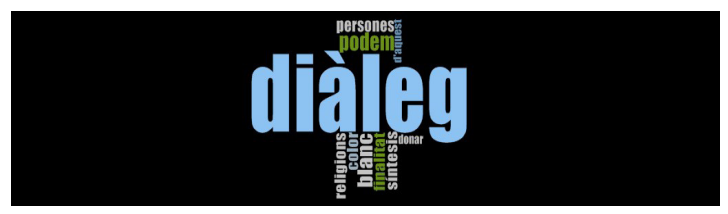

Gráfico 1. Marca de nube general con las narrativas significativas de la definición sobre la categoría diálogo interreligioso e intercultural en los expertos académicos. Elaboración propia con base en información arrojada por el software Nvivo ${ }^{2}$

El diálogo interreligioso supone la predisposición a repensar las ideas propias a la luz de las de los demás, dejando abierta la posibilidad de enriquecimiento y transformación mutuos entre los participantes (Santiago \& Corpas, 2012):

"Otra cosa es que después del primer paso, de este reconocimiento al otro, el segundo paso es que después de conocernos, ¿nos podemos fecundar? y también ¿nos podemos transformar?" (Ent. 4, Grupo 3, ref. 3). 
Ésta es la capacidad de dejarse interpelar por el otro sin dejar de ser uno mismo/a, y avanzar hacia el blanco como suma de todos los colores, hacia una síntesis integradora de orden superior que no asimila ninguna parte y es más que la suma de todas ellas:

"El color blanco no es el violeta, ni el verde, ni el rojo... El color blanco es otro color, síntesis superior de cada uno de los colores. Las mezclas son situaciones intermedias en cambio la síntesis es el blanco. Entonces, ¿vamos hacia el blanco? Mirándolo místicamente yo creo que si" (Ent. 5, Grupo 3, ref. 5).

Por lo tanto, se trata de una narrativa que comparte la definición sobre el diálogo interreligioso aportada por la UNESCO (2015:80) en la cual la horizontalidad está en sintonía con "la igualdad" y que lo entiende como "intercambio de palabras y escucha recíproca que compromete en pie de igualdad los creyentes de diferentes tradiciones religiosas" pero situándolo en el plano personal. También es coherente con los elementos conceptuales del propio Libro Blanco sobre el Diálogo Intercultural (Unión Europea 2006), donde se define como un proceso que implica el intercambio respetuoso y abierto de puntos de vista entre individuos y grupos de diferentes procedencias y con legados culturales, religiosos y lingüísticos diferentes, sobre la base del mutuo entendimiento y respeto.

Desde la Administración Pública se hace referencia al diálogo intercultural para definir el diálogo interreligioso: el diálogo religioso va más allá del diálogo intercultural, pero es una forma de éste. Como se vislumbra en la marca de nube (Gráfico 2) las categorías interreligioso e intercultural emergen justo alrededor de la palabra diálogo que es la más utilizada en el relato. Se remite a la relación entre cultura y religión para definir el diálogo intercultural e interreligioso. La diversidad cultural se manifiesta de manera significativa en la diversidad religiosa y de convicciones y se debe vehicular positivamente a través del diálogo entre culturas y religiones:

"entiendo que el diálogo interreligioso puede ser una forma de diálogo intercultural, pero va un poco más allá (...) porque también pueden ser personas que sean de la misma cultura y que tengan religiones diferentes" (Ent 1, Grupo 1, ref. 1-2).

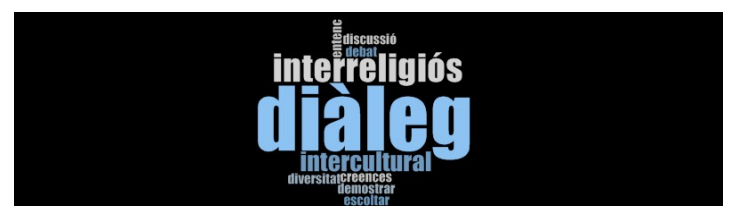

Gráfico 2. Marca de nube general con las narrativas significativas de la definición sobre la categoría diálogo interreligioso e intercultural en la Administración. Elaboración propia con base en información arrojada por el software Nvivo

El siguiente mapa ramificado también evidencia que la definición y el significado de este diálogo en la narrativa de la Administración están íntimamente relacionados con el valor de la escucha y el entendimiento con el otro sobre la diversidad y las creencias diferentes. Aunque el conocimiento del otro por sí mismo no garantiza el diálogo, es un requisito inevitable. $Y$, si, tal como señalan Moliner y Aguilar (2010), este conocimiento se ofrece con espíritu crítico y con la empatía necesaria tiene todas las garantías de favorecer una cultura del diálogo que posibilite la vida en común y el intercambio cultural:

"Vivimos en una sociedad diversa y plural y (...) por lo tanto, tenemos que convivir en esta diversidad y tenemos que aprovechar esta diversidad para enriquecernos, para hacer una sociedad más cohesionada" (Ent 1, Grupo 1, ref. 2-3). 


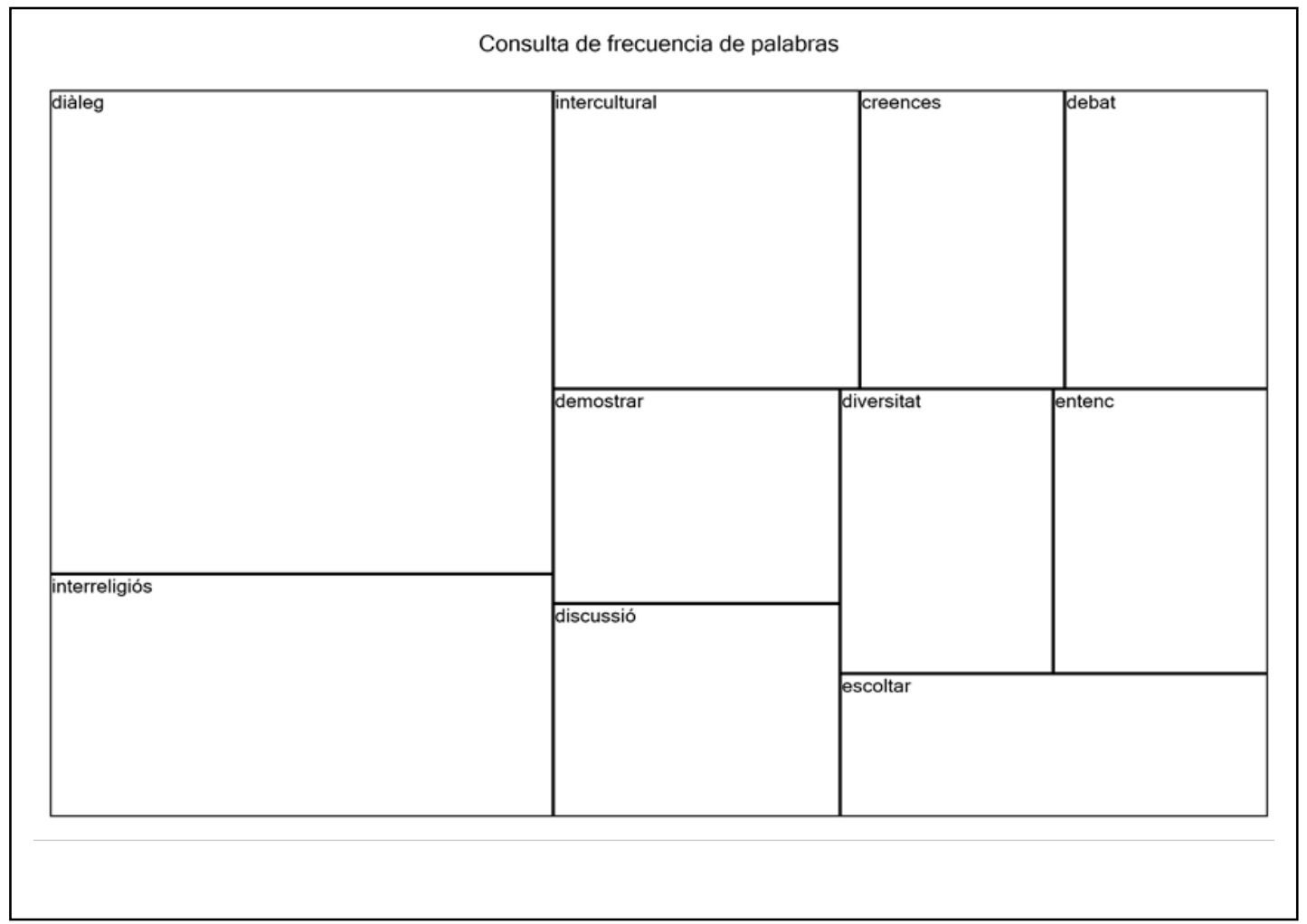

Gráfico 3. Mapa ramificado general con las narrativas significativas sobre la categoría diálogo interreligioso e intercultural en la Administración.

Las entrevistas de los profesionales de las entidades ofrecen un relato del diálogo interreligioso e intercultural no referido tanto a la finalidad como a los grandes beneficios del proceso en sí mismo: mejor autoconocimiento, relativización del propio punto de vista y capacidad de autocrítica para abrirse a los demás y, en última instancia, beneficio social para una mejor ciudadanía. En palabras de Torradeflot (2012), los principios del diálogo interreligioso se orientan a desarrollar la capacidad de escucha, respetar la diversidad de creencias, identificar la experiencia religiosa común, la apertura a la diferencia, dando prioridad a los derechos humanos y la democracia y aportando soluciones a los problemas con una ciudadanía crítica y participativa. $\mathrm{Y}$ así mismo lo expresaba una experta del mundo de las entidades entrevistada:

"Pero, cuando hacemos un esfuerzo de relación surge un contacto entre las partes y esto nos permite disfrutar de un conocimiento mutuo que facilita cualquier gestión y nos convierte en una sociedad más civilizada y organizada, sin dudarlo". (Ent. 2, Grupo 2, ref.r).

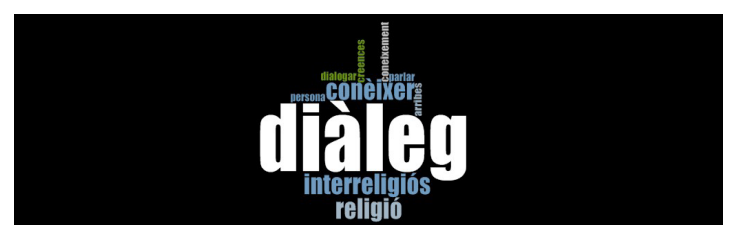

Gráfico 4. Marca de nube general con las narrativas significativas de la definición sobre la categoría diálogo interreligioso e intercultural en los profesionales de las entidades. Elaboración propia con base en información arrojada por el software Nvivo.

Cuando el diálogo interreligioso es de proximidad, además de ayudar a prevenir y resolver conflictos interreligiosos, enriquece los procesos de participación ciudadana, creando las condiciones que permiten la solidaridad y la justicia (Torradeflot, 2011).

Estos resultados confirman y se ajustan a los tres sentidos en que la UNESCO (2015) entiende que se practica y se fomenta el diálogo interreligioso:

a) para vivir en un espíritu de apertura y cohesión, un reto social que en el discurso de la Administración se reivindica como muy relevante para la gestión de la diversidad cultural y religiosa.

b) para colaborar con vistas al desarrollo integral de personas de diferentes religiones $y$ 
que, desde la visión de Pániker (2003), debe producirse especialmente en el sentido místico entre las raíces espirituales de cada tradición y la identidad. El miedo a perder la identidad en algunos grupos y comunidades o el natural deseo de integridad, preservación y continuidad desde una percepción asimétrica, etnocéntrica y de superioridad puede derivar en un cierre, exclusión e incluso, odio. El diálogo interreligioso tiene el reto de encontrar la forma de romper estas barreras (Torradeflot, 2011). Según uno de los expertos académicos entrevistados, superar las barreras de la diferencia implica aprovechar las oportunidades de aproximación naturales para generar espacios comunes y promover el diálogo:

"Aprovechar las oportunidades, nos tenemos que conocer todavía más, somos muy reticentes para acercarnos a los demás. Trabajar más las sensibilidades que facilitan el encuentro y la naturalidad de éste porque se genera un espacio común." (Ent 5, Grupo 3, ref. 2).

c) para comprender más profundamente las diferentes herencias religiosas y apreciar los valores espirituales. El conocimiento de lo que es común permite aprender y apreciar mejor las diferencias. Desde esta perspectiva, el diálogo interreligioso comprende el trabajo de la dimensión espiritual de la persona, y no sólo en el aspecto de la religiosidad:

“No sé cómo decirlo; las creencias religiosas o las adopciones y prácticas de unas creencias religiosas pueden ir y estar muy al margen del entorno" (Ent 1, Grup 1, ref. 1)

"Yo creo que el hecho de conocer personas que piensan diferente a ti, ayuda a cuestionar la propia fe o tu forma de vivir y por lo tanto, creo que es muy importante sobre todo por este conocimiento que te acerca a las persones que tienen otras formas de vivir, de creer... “(Ent. 1, Grupo 2, ref. 1)

"El diálogo es un paso siguiente a la mera constatación de la pluralidad y el diálogo incluye un interés por el otro y una capacidad de dejarse interpelar por el otro". (Ent. 5, Grupo 3, ref. 7).

El diálogo interreligioso en sentido amplio no se puede concebir sin la inclusión del diálogo entre el ateísmo, el agnosticismo, la diversidad religiosa, las creencias y las espiritualidades diversas.

\section{Los elementos que favorecen el diálogo interreligioso e intercultural}

En cuanto a los elementos relevantes para favorecer el diálogo interreligioso e intercultural, también se observan patrones diferentes según los colectivos entrevistados tal y como evidencia la Tabla 3. Así, en el relato de los expertos académicos el peso de la información codificada a partir de esta categoría es significativamente superior sobre todo en comparación con la narrativa de la Administración cuya relevancia es mínima si consideramos los porcentajes de esta columna (véase 3.2. Elementos que favorecen el diálogo intercultural e interreligioso).

A partir de la narrativa de los expertos del mundo académico, de acuerdo con el conteo de frecuencia de palabras de los sujetos entrevistados, actualmente el diálogo interreligioso es un elemento relevante para la cohesión social, coherente con la necesidad de nueva sensibilidad interreligiosa (Torradeflot, 2011) y la vivencia y existencia de la diversidad religiosa en nuestro contexto (Gráfico 5).

Consulta de frecuencia de palabras

\begin{tabular}{|c|c|c|c|c|c|c|}
\hline \multirow[t]{2}{*}{ religiosa } & \multirow[t]{2}{*}{ |diàleg } & \multirow[t]{2}{*}{ Feligions } & diversitat & sentit & societat & Interreligiós \\
\hline & & & totes & encara & islam & \\
\hline
\end{tabular}

Gráfico 5. Mapa ramificado general con las narrativas significativas sobre la categoría elementos que favorecen el diálogo interreligioso e intercultural en los expertos académicos. 
Entre los aspectos destacados por este colectivo para fomentar el diálogo intercultural e interreligioso se insiste en tres ejes nucleares:

\section{A) DESDE LA COMUNIDAD}

- El conocimiento mutuo y el fomento del contacto desde la base, para superar las barreras a la diferencia, aprovechando las oportunidades de contacto naturales para generar espacios comunes para el diálogo:

"De todas maneras hay que trabajar para que la realidad se exprese, se debe generar todo tipo de plataforma desde la plástica hasta lo social" (Ent 5, Grupo 3, ref. 2).

\section{B) DESDE LAS ENTIDADES SOCIALES}

- La competencia comunicativa, el saber explicarse bien, especialmente por parte de los líderes religiosos o representantes de entidades religiosas para favorecer el encuentro intercultural e interreligioso:
“...incapacidad por parte de muchos de los líderes religiosos y de los representantes de las comunidades religiosas en explicarse y es un problema comunicativo, no que no sepan quienes son o que hacen, sino que no conocen - traducen para el público en general para explicar quiénes son" (Ent 2, Grupo 3, ref. 2).

También es importante preparar los medios de comunicación para que sepan explicar, reconocer y hacer inteligible el hecho religioso y la diversidad confesional, pues existe una enorme ignorancia al respecto en la opinión pública. El riesgo de no darse este requisito es la construcción de un imaginario colectivo repleto de incorreciones, estereotipos y prejuicios.

- La educación formal para dar a conocer y reconocer la diversidad cultural y religiosa. Concretamente, un experto académico argumenta que la falta de formación en religión en los centros educativos fomenta el desconocimiento de los propios referentes, dificultando el diálogo intercultural e interreligioso:

"Pero estos no han recibido nada de esto, normalmente, de las públicas y algunas de las privadas ignoran absolutamente todo esto, hace que no sean capaces de comprender el mundo en el que viven y el mundo en el que han vivido" (Ent 3, Grupo 3, ref. 7).

\section{C) DESDE LA POLITICA}

- El papel de la Administración pública y el gobierno para favorecer el diálogo interreligioso, solicitando explícitamente la independencia de las organizaciones o departamentos especializados respecto a los partidos o voluntades políticas:

“...soy un poco pesimista porque creo que las decisiones que se han ido tomando en los últimos 14 o 15 años son de carácter político, no son de carácter educativo. Esto parece un pingpong, partido que gobierna irá hacia un lado y hacia el otro y no apuntamos al tema religioso que es un tema arraigado en la cultura ... es un tema complicado" (Ent 2, Grupo 3, ref. 3).

Desde la Administración Pública se señala la importancia social y comunitaria, muy cercana a discursos que plantean la necesidad de diálogo entre todos los agentes activos del territorio (comunidades religiosas, vecinos, comerciantes, etc.) para favorecer el diálogo:

"La prioridad es poder abrir cada día la puerta del centro de culto, si lo tienen, y hacer sus actividades $y$ en ocasiones con esto, sus recursos quedan un poco agotados ino?" (Ent 1 , Grupo 1, ref. 1).

Hay que crear instancias que favorezcan este encuentro y convoquen a todas estas entidades a participar en el mismo: el diálogo de base que es el de las asociaciones.

Finalmente, desde la perspectiva de las entidades, como se vislumbra en la marca de nubes siguiente (Gráfico 6), la multiconfesionalidad exige reconocer y valorar positivamente las diferencias para establecer puentes de diálogo y crear un imaginario colectivo sobre el hecho religioso más abierto a la tradición católica tradicional.

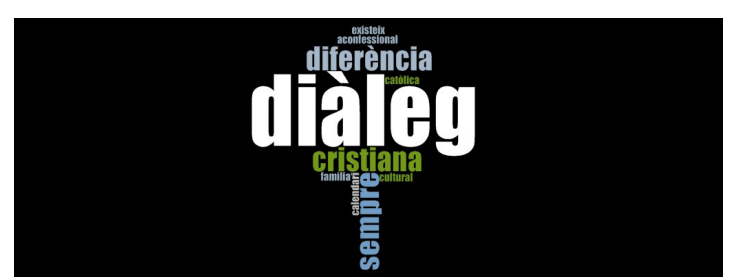

Gráfico 6. Marca de nube general con las narrativas significativas de la definición sobre la categoría elementos que favorecen el diálogo interreligioso e intercultural en los profesionales de las entidades. Elaboración propia con base en información arrojada por el software Nvivo. 
Una de las personas entrevistadas del mundo de las entidades propone promover la participación de las instituciones religiosas constituyéndolas como interlocutoras de las iniciativas para el diálogo interreligioso:

"Una institución pública firme, una formación universitaria, unos grupos de diálogo que realmente se tuvieran en cuenta a la hora de resolver los temas el que intervengan los colectivos religiosos aumentando su presencia en los grupos de diálogo" (Ent 4, Grupo 2, ref. 3).

\section{Discusión y conclusiones}

Aunque desde la Administración, academia y entidades se define el diálogo interreligioso de forma diferenciada, su conceptualización se complementa. La primera pone el acento en la relación entre la cultura y la religión siendo el valor de la escucha y el entendimiento, otros dos elementos importantes para el diálogo. La relación entre religión y cultura ha sido ampliamente discutida desde la antropología, la sociología y la filosofía (Morgan \& Sandage, 2016), sugiriendo una mediación cultural de la experiencia religiosa. Por su parte, las personas académicas entrevistadas aterrizan la definición en el plano individual puesto que el diálogo se establece entre personas. Estos interlocutores deben reconocerse mutuamente y dejarse interpelar por el otro; situación que, va a permitir profundizar en la propia identidad: pertenecer a un grupo religioso se percibe como una dimensión social o colectiva (Huddy, 2003). Finalmente, las entidades participantes manifiestan los beneficios del diálogo interreligiosos tanto para la persona como para la sociedad promoviendo y facilitando una ciudadanía más crítica y participativa, en línea con los planteamientos de Torradeflot (2012).

Los participantes relatan también los grupos de profundización en intercambio espiritual, de estudio y profundización y el diálogo de vida como tipos diferentes de diálogo. Los grupos de diálogo de representación institucional no son nombrados explícitamente por la Administración, pero veladamente muestran la importancia de crear estas instancias. Son las entidades quienes demandan esta interlocución.

Finalmente, destacar el papel de la educación en el conocimiento y reconocimiento de la diversidad cultural y religiosa, tal como indican las personas entrevistadas. Conocer la diversidad y la profundidad de las tradiciones religiosas y de sabiduría y de los propios referentes permite el enriquecimiento personal y el conocimiento mutuo, facilita el diálogo intercultural e interreligioso, y previene actitudes integristas. Habría que ofrecer una formación básica en el conocimiento de la diversidad religiosa y las convicciones no religiosas como las visiones ateas, agnósticas o indiferentes. Morgan y Sandage (2016) ponen de manifiesto como la rigidez mental va ligada a los estereotipos, prejuicios e incluso, procesos de discriminación por razones culturales o religiosas. Por tanto, el conocimiento de la propia espiritualidad y la diversidad cultural y religiosa puede ser un inicio de superación de estas tendencias discriminatorias.

Considerando los sistemas de la teoría ecológica de Bronfenbrener (1992), los datos obtenidos (véase Tabla 3 con el peso que adquiere la categoría 3.3 sobre las iniciativas comunitarias y/o políticas actuales existentes en el relato de los tres colectivos) aportan luz para construir una propuesta de gestión de la diversidad religiosa para el fomento del diálogo intercultural e interreligioso en el espacio público.

El microsistema es el entorno diario de la persona, en donde se puede producir el diálogo de vida. La familia constituye uno de los microsistemas principales cuyo ámbito puede ser definido como privado y en el que se vive y/o comparte una religión, creencia, convicción espiritual o de vida. La escuela es un contexto formal con diversidad religiosa que debería aprovechar la oportunidad para fomentar este diálogo de vida en sus espacios. Los espacios informales como el patio de la escuela son un lugar de contacto natural para favorecer el conocimiento mutuo tal como indican Torradeflot (2012) y algunos de los participantes cuando describen el eje de la comunidad para fomentar el diálogo interreligioso. En el espacio formal, el estudio de las religiones permite comprender la diversidad religiosa a la vez que los propios referentes de cada niño y niña que explican las tradiciones y creencias de sus familias, aspecto señalado tanto por los expertos académicos entrevistados como por la literatura. Así, Moliner y Aguilar (2010) relatan que si este conocimiento se ofrece con espíritu crítico y con la empatía necesaria, tiene todas las garantías de favorecer una cultura del diálogo para la vida en común y el intercambio cultural. Elósegui (2009) apuesta por una educación intercultural con una sólida carga pedagógica en el ámbito de la educación en valores. En concreto, es un conjunto básico de valores densos: desde la hospitalidad y el reconocimiento hasta la solicitud y la ineludible solidaridad responsable con cualquier otro. Demanda con urgencia una educación ética radical, capaz de superar todo tipo de egocentrismo, posibilitando así una convivencia intercultural enriquecedora.

En el mesosistema, relación entre los microsistemas, se pueden establecer grupos o iniciativas 
de diálogo de reconocimiento mutuo. La escuela constituye, a su vez, el espacio idóneo para la creación de vínculos con las organizaciones religiosas y no religiosas y para el encuentro entre alumnado con diferentes actitudes ante la religión (Weisse, 2009; Jackson 2015), formando así un mesosistema que apoya el reconocimiento mutuo. Moliner y Aguilar (2010) y Weisse (2009) proponen la participación de expertos de las diferentes comunidades para asesorar los procesos de creación y desarrollo de currículum y materiales educativos. La escuela puede organizar distintas actividades de reconocimiento mutuo, participando no solamente su alumnado sino también las familias, para descubrir al otro: merienda con los platos típicos explicando su relación con sus creencias, exposición de alguna expresión plástica de las familias sobre el arte religioso, etc. Son espacios que fomentan la identidad de cada persona y colectivo con creencias diferentes, a la vez que confronta la propia identidad con la del otro. El mesosistema permite establecer diálogos desde la naturalidad, la proximidad del otro, la alteridad al compartir espacios públicos.

El exosistema está representado por lo grupos de estudio, de diálogo espiritual y de cohesión social y mediación que describe Torradeflot (2011) puesto que influyen de manera indirecta en la vida de las personas. Son diálogos promovidos desde las entidades, tal como lo expresan ellas mismas en la investigación, pero que necesitan de la interlocución de la Administración que no se posiciona de forma explícita en la entrevista realizada. La potencialidad de estos grupos reside en su contribución para eliminar prejuicios y en el fomento de la cohesión de los municipios y barrios, entre otros.

Finalmente, el macrosistema está conformado por el sistema de creencias de la sociedad; en nuestro caso, sobre la religión, la diversidad religiosa, el diálogo interreligioso. Es de vital urgencia adoptar políticas en asuntos religiosos que permitan aprovechar la diversidad del patrimonio cultural y religioso, tal como Buades y Vidal (2007) conceptualizan la credodiversidad reclamando la autonomía política y la cooperación pública de las religiones en la búsqueda de lo común. Es por ello, que distintos participantes del estudio en el eje de la política de los resultados reclaman la independencia tanto de las entidades como del Departament d'Afers Religiosos de los partidos o voluntades políticas.

Una de las limitaciones de este estudio puede situarse en la composición de la muestra en el sentido de que no hay una representación de todas las trece confesiones religiosas que conviven en Cataluña. Si bien es cierto que se han entrevistado a dos teólogos y a una persona de la confesión islámica que representan solamente dos creencias religiosas, también lo es que la sociedad catalana, según el Mapa religiós de Catalunya (2014), está compuesta en su mayoría por personas católicas (58\%), seguidas por las que se consideran ateas (16\%) y agnósticas (12\%), siendo la religión islámica $(4,8 \%)$, la mayoritaria entre las confesiones minoritarias. También, hay que tener en cuenta que los primeros pasos hacia el diálogo interreligioso en Cataluña se han producido a partir de expertos que bien provienen de una tradición católica o bien de un eclecticismo de distintas tradiciones religiosas.

Las líneas de futuro sobre el diálogo interreligioso en Cataluña deberían dar la palabra a todas las confesiones religiosas para no tener una perspectiva excluyente y preeminente de una religión sobre las otras, en nuestro caso, del catolicismo sobre las otras doce confesiones. Ello es uno de los elementos que puede favorecer la eliminación de los procesos de radicalización, especialmente de jóvenes, que estamos viviendo en la Unión Europea.

\section{Notas}

1 Resultados parciales de la investigación subvencionada por la Direcció General d’Afers Religiosos de la Generalitat de Catalunya. En el caso de ser aceptado el artículo se indicará título y código del proyecto.

2 Las marcas de nube y los mapas ramificados que se presentan en el artículo mantienen las palabras originales de las entrevistas, efectuadas en catalán. Por ello los términos corresponden a esta lengua. Entre los más citados, ofrecemos la traducción de: diàleg (diálogo), interreligiós (intereligioso), diversitat (diversidad), religió (religión), conèixer (conocer). 


\section{Referencias bibliográficas}

Aaroe, L. (2012). Does tolerance of religion in the public space depend on the salience of the manifestation of religious group membership? Political Behavior, 34, 585-606.

Álvarez, J.L., \& Essomba, M.A. (2012). Dioses en las aulas. Educación y diálogo interreligioso. Barcelona: Graó.

Bauman, Z. (2002). Modernidad líquida: México: S.L. Fondo de cultura económica de España.

Bowen, J. R. (2008). Why the French don't like headscarves. Princeton: Princeton University Press.

Brie, M. (2011). Ethnicity, Religion and Intercultural Dialogue in the European Border Space. Debrecen/Oradea: Editura Universitatii din Oradea.

Bronfenbrenner, U. (1992). Ecological systems theory. In R. Vasta (Ed.), Six theories of child development: Revised formulations and current issues (pp. 187-248). Philadelphia: Jessica Kingsley

Buades, J., \& Vidal, F. (2007). Minorías de lo Mayor. Religiones minoritarias en la Comunidad Valenciana. Barcelona: Icaria.

Cabrera, F.A. (2011) Técnicas e instrumentos de evaluación: una propuesta de clasificación. [En línea] REIRE, Revista d'Innovació i Recerca en Educació, Vol. 4, núm. 2, 112-124. http://www.raco.cat/index.php/REIRE

Corbí, M. (2007). Hacia una espiritualidad laica: sin creencias, sin religiones, sin dioses. Barcelona: Herder.

Creswell, J.W. (2003). Research design: Qualitative, quantitative, and mixed approaches. Thousand Oaks, CA: Sage.

Creswell, J. W., \& Plano Clark, V. L. (2011). Designing and conducting mixed methods research. (2nd ed.). Thousand Oaks, CA: Sage.

Dietz, G. (2008). La educación religiosa en España: ¿̇contribución al diálogo intercultural o factor de conflicto entre religiones?, Estudios sobre las Culturas Contemporaneas, XIV (28), 11-46.

Direcció General d'Afers Religiosos. (2014). Mapa religiós de Catalunya. Generalitat de Catalunya. http://governacio. gencat.cat/ca/pgov_ambits_d_actuacio/pgov_afers-religiosos/pgov_serveis/pgov_mapa_religions/.

Dupuis, J. (2002) El cristianismo y las religiones. Del desencuentro al diálogo. Sal Terrae.

Elósegui, M. (2009). Políticas públicas y educación intercultural. A M. Esteban (Coord.). El diálogo intercultural en España: un requisito de la educación y cultura de Paz. Madrid: Ministerio de Educación.

Flick, U. (2004). Introducción a la Investigación Cualitativa. Madrid: Morata.

Gibbs, G. (2012). El análisis de datos cualitativos en investigación cualitativa. Madrid: Morata.

Hozu, J., \& Frunza, S. (2013). Religious affiliation and social action in the public space. Revista de cercetare si interventie sociala, 43, 240-254.

Huddy, L. (2003). Group identity and political cohesion. In D.O. Sears, L. Huddy, \& R. Jervis (Eds.), Oxford handbook of political psychology (511-558). Oxford: Oxford University Press.

Jackson, R. (2015). Señales-Políticas públicas y prácticas para la enseñanza de las religiones y las cosmovisiones no-religiosas en la educación intercultural. Estrasburgo: Editorial del Consejo de Europa.

Javier, M. (2014) El diálogo interreligioso acerca a las distintes religiones a la modernidad. Tendencias 21. Tendencias de las religiones. http://www.tendencias21.net/El-diálogo-interreligioso-acerca-a-las-distintas-religiones-en-la-modernidad_a37996.html

Kettell, S. (2009) On the public discourse of religion: An analysis of Christianity in the United Kingdom. Politics and Religion, 2, 420-443.

Maykut, P., \& Morehouse, R. (1999). Investigación cualitativa: una guía práctica y filosófica. Barcelona: Hurtado.

Melloni, J. (2003). El Uno en lo múltiple. Aproximación a la diversidad y la unidad de las religiones. Santander: Sal Terrae.

Moliner, A., \& Aguilar, N. (2010). Les tradicions religioses en els llibres de text. Barcelona: Montflorit Edicions i Assessoraments S.L.

Morgan, J., \& Sandage, S. J. (2016). A developmental model of interreligious competence. A conceptual framework. Archive for the Psychology of Religion, 38, 129-158.

Pániker, S. (2003). El diàleg indispensable. Pau entre les religions. Barcelona: Edicions 62.

Panikkar, R (2005) De la Mística. Experiencia plena de la Vida. Barcelona: Herder

Pániker, S. (2010). Pluralisme i interculturalitat, Cultures i religions en diàleg. Barcelona, Omnia.

Prieto, M.D. (2014) El pluralismo y la diversidad enriqueceran las tradiciones religiosas. Revista electrónica de ciencia, tecnología, sociedad y cultura. http://www.tendencias21.net/el-pluralismo-y-la-diversidad-enriqueceran-las-tradiciones-religiosas_a37465.html

Santiago, M., \& Corpas, C. (2012). Bases para el desarrollo de buenas prácticas de educación para la convivencia en la diversidad cultural y religiosa. En, J. L. Álvarez; M. A. Essomba (coords.), Dioses en las aulas. Educación y diálogo interreligioso (pp. 123-143). Barcelona: Graó.

Tamayo, J.J. (2012) Otra teología es posible. Interculturalidad, pluralismo religioso y feminismos. Barcelona: Herder. Tashakkori, A., \& Teddlie, C. (Eds.). (2010). Sage handbook of mixed methods in social \& behavioral research. Sage. 
Torradeflot, F. (2011). Religiones y pluralismo. Las vías del diálogo interreligioso en España. Madrid: Observatorio del Pluralismo Religioso en España.

Torradeflot, F. (coord.) (2012). Catalunya i el diàleg interreligiós. Aportacions innovadores al diàleg interreligiós des dels valors de les nacions unides. Barcelona: Associació UNESCO per al diàleg interreligiós.

UNESCO (2005). The Rabat Commitment Conclusions and Recommendations of the Rabat Conference on Dialogue among Cultures and Civilizations through Concrete and Sustained Initiatives Rabat, Morocco. Acceso en: http:// www.unesco.org/dialogue/rabat/commitment.html.

UNESCO (2015) Diccionari de religions. Barcelona: Generalitat de Catalunya. Departament de Governació i Relacions Institucionals.

Unión Europea (2006) Libro Blanco sobre el Diálogo Intercultural. Decisión n01983/2006/CE del Parlamento Europeo y del Consejo del 18 de diciembre.

Vilà, R., Burguet, M., Escofet, A. \& Rubio, M.J.(2015) Hacia el dialogo intercultural e interreligioso: necesidades normatives para una gestión de la diversidad religiosa en el espacio público. Comunicación presentada en el $\vee$ Congreso REPS: Desigualdad y democracia: Políticas públicas e innovación social. Barcelona. 5 y 6 de febrero.

Weisse, W. (coord.) (2009). Religión en educación: Contribución al diálogo. Sugerencias del proyecto de investigación REDCo para la política. Comisión Europea. Acceso en: https://www.uni-hamburg.de

\section{CÓMO CITAR ESTE ARTÍCULO}

Sabariego, M., Vilà, R., \& Freixa, M. (2018). El diálogo interreligioso en el espacio público: retos para los agentes socioeducativos en Cataluña. Pedagogía Social. Revista Interuniversitaria, 32 151-166. DOI:10.7179/PSRI_2018.32.11

\section{DIRECCIÓN COMPLETA DE LOS AUTORES}

MARTA SABARIEGO PUIG. Universidad de Barcelona. Facultad de Educación. Departamento Métodos de Investigación y Diagnóstico en Educación. Despacho 2O2. Passeig de la Vall d'Hebron, 171. Edifici Llevant-2a planta. 08035 Barcelona. Tel.: (o034) 93.403.50.10. E-mail: msabariego@ub.edu

RUTH VILÀ BAÑOS. Universidad de Barcelona. Facultad de Educación. Departamento Métodos de Investigación y Diagnóstico en Educación. Despacho 264. Passeig de la Vall d'Hebron, 171. Edifici Llevant-2a planta. O8035 Barcelona. Tel.: (0034) 93.403.52.13. E-mail: ruth_vila@ub.edu

MONTSERRAT FREIXA NIELLA. Universidad de Barcelona. Facultad de Educación. Departamento Métodos de Investigación y Diagnóstico en Educación. Despacho 204. Passeig de la Vall d'Hebron, 171. Edifici Llevant-2a planta. 08035 Barcelona. Tel.: (0034) 93.403.50.19. E-mail: mfreixa@ub.edu 


\section{PERFIL ACADÉMICO}

MARTA SABARIEGO PUIG. Doctora en Filosofía y Ciencias de la Educación. Profesora titular de Universidad del Departamento de Métodos de Investigación y Diagnóstico en Educación (MIDE) de la Facultad de Educación de la Universitat de Barcelona. Miembro del Grupo de Investigación en Educación Intercultural (GREDI). Los proyectos más recientes en su trayectoria académica se han centrado en el desarrollo de metodologías participativas para promover la convivencia y la inclusión social en contextos comunitarios y multiculturales, en calidad de coordinadora (Ministerio de Educación, Política Social y Deporte- Fundación Europea Sociedad y Educación, 2009; Fundación InteRed, 2010; Programa Estatal de Investigación, Desarrollo e Innovación Orientada a los retos de la sociedad- EDU2013-46704-R) y como miembro del equipo investigador (Ministerio de Educación y Ciencia- EDU2008-00332/EDUC; ARAF1 00045; AGAUR, 2014-RELIGooo19 y AGAUR, 2015-RELIGOOOO7).

RUTH VILÀ BAÑOS. Doctora en Psicopedagogía. Profesora en el departamento de Métodos de Investigación y Diagnóstico en Educación de la Universitat de Barcelona. Forma parte del grupo de investigación en Educación Intercultural (GREDI). Es miembro del Consejo de Dirección del Instituto de Investigación en Educación (IRE). Ha participado en investigaciones sobre competencias interculturales y diálogo intercultural e interreligioso. Ha coordinado dos proyectos subvencionados por Asuntos Religiosos de la Generalitat de Cataluña: Gestionar la diversidad religiosa y cultural a la educación desde el diálogo intercultural e interreligioso (2014RELIGooo19) y Las entidades y los centros de Secundaria ante la diversidad religiosa (2015RELIG00007). ResearchGate: https://www.researchgate.net/profile/Ruth_Vila.

MONTSERRAT FREIXA NIELLA. Doctora en Filosofía y Ciencias de la Educación. Profesora titular de Universidad del Departamento de Métodos de Investigación y Diagnóstico en Educación (MIDE) de la Facultad de Educación de la Universitat de Barcelona. Ha sido secretaria de Departamento, jefe de estudios de Educación social y vicedecana académica de la Facultad de Pedagogía. Miembro del grupo de investigación Trals, consolidado por la Generalitat de Catalunya. Su trayectoria investigadora se ha desarrollado fundamentalmente vinculada a proyectos sobre las transiciones, el abandono escolar y universitario y la orientación universitaria. Las investigaciones más recientes se centran, por una parte, en la Transiciones y trayectorias de acceso a los estudios de Másteres universitarios (MCOC, EDU-2016-80578-R) y por otra, en la diversidad religiosa y el diálogo interreligioso en el ámbito de la educación (AGAUR, 2014-RELIGo0019 y AGAUR, 2015-RELIG00007). 